\title{
EDITORIAL
}

\section{LA NECESIDAD DE UNA MEJORA CONTINUA DE LAS ESTADÍSTICAS DE MORTALIDAD}

\author{
Andreu Segura Benedicto (1) y Jose Miguel Mata de la Torre (2). \\ (1) Institut Universitari de Salut Pública de Catalunya. \\ (2) Centro Nacional de Información Sanitaria. Instituto de Salud Carlos III. Ministerio de Sanidad y Consumo.
}

Desde que, a mediados del siglo XVII, John Graunt publicara sus observaciones naturales y políticas sobre las cuentas de mortalidad, referidas a los registros parroquiales de Londres, el estudio de las defunciones constituye un elemento básico para los análisis demográficos y sanitarios. Aunque, para ser históricamente precisos, debe señalarse que el trabajo pionero de Graunt fue el germen de un proceso que requirió un par de siglos para fructificar, hasta que William Farr iniciara la publicación anual de las estadísticas de mortalidad en 1837. Tanta importancia tuvo este hecho para la salud pública que algunos autores, como Lilienfeld, consideran que si la moderna epidemiología se desarrolló primero en Inglaterra fue por esa iniciativa que no se produciría sino más tardíamente en Francia, a pesar de la importancia de la figura de Pierre Alexander Louys, el creador de la medicina numérica ${ }^{1}$.

En España la semilla de Graunt también tuvo su influencia y algunos médicos, como Salvá i Campillo, reivindicaron la construcción de los análisis estadísticos de la mortalidad $^{2}$, aunque hubo que esperar hasta finales del XIX para que se creara el Registro Civil y, en la práctica, dispongamos de series estadísticas de mortalidad desde 1900.

Precisamente, en este año se plantea la introducción de la décima revisión de la clasificación internacional de enfermedades y causas de defunción y tal vez sea ésta una oportunidad para recuperar desde sanidad una iniciativa que, conjuntamente con el Instituto Nacional de Estadística (INE), se llevó a cabo hace ya casi diez años.

Un nutrido grupo de trabajo en el que colaboraron estadísticos, informáticos y epidemiólogos llevó a cabo la revisión de los boletines estadísticos de defunción que se introdujeron por el INE en 1975 y que consiguieron en su momento, una sustantiva mejora de la calidad de las estadísticas oficiales de mortalidad. La información sobre las estadísticas vitales, recogida mediante los formularios de 1975 se trató, desde entonces, en soporte informático. de manera que las posibilidades de análisis se incrementaron notablemente.

Sin embargo, el grupo de trabajo propuso algunas modificaciones en los boletines, desde la denominación misma - que debería ser la de certificado sanitario de nacimiento y de certificado médico de defunción-y la inclusión de un nuevo impreso, el certificado médico de defunción perinatal, para incrementar la cobertura de las defunciones ocurridas en el período perinatal, el cual es objeto de subregistro ${ }^{3}$.

Las modificaciones introducidas tenían que ver, básicamente, con la adaptación del apartado de causas de muerte a los estándares internacionales y con la facilitación de la 
codificación para el tratamiento informatizado de las variables que en su momento se consideró podían ser recogidas con la máxima exactitud posible.

Han pasado los años y seguimos disponiendo de los mismos impresos de recogida de datos que se introdujeron en 1975. Pero durante estos casi treinta años se han producido algunos cambios notables, entre los que cabe destacar la colaboración de las Comunidades Autónomas en la recogida y la validación de los datos, y hasta en la codificación y análisis para cada uno de los territorios autonómicos. Lo que, en conjunto, ha supuesto un incremento de la puntualidad de las publicaciones referidas a la mortalidad y un mayor uso en las tareas de planificación sanitaria, particularmente en el diseño de los planes de salud de las comunidades autónomas.

Del mismo modo se han establecido programas de mejora de la calidad de la información, mediante la verificación sistemática de la exactitud de los boletines y, también, mediante el establecimiento de actividades específicas de formación de los médicos certificadores.

Tal vez vale la pena recordar que la introducción de este tipo de actividades en España, salvo error u omisión, se remonta a finales de los años setenta y principios de los ochenta cuando, con ocasión del inicio del programa CRONICAT y de su incorporación al programa MONICA de la OMS, se constató la necesidad de mejorar los datos referidos a la mortalidad por enfermedades crónicas. A partir de esta constatación se introdujo en el programa de la asignatura de Medicina Preventiva en la licenciatura de Medicina de la Universidad Autónoma de Barcelona, un seminario específico sobre la correcta certificación médica de las causas de defunción.

Pero todavía más relevante fue el desarrollo de esta iniciativa formativa por parte de las administraciones sanitarias, de manera que el programa se extendió a los médi- cos certificadores, priorizando las convocatorias, al menos por parte de algunas Comunidades Autónomas, a los facultativos que más boletines de defunción cumplimentaban y los que más problemas presentaban. En este sentido el Ministerio de Sanidad promovió la realización de diversos seminarios, de forma que la experiencia se pudiera generalizar.

Este tipo de actividad se sigue llevando a cabo por parte de algunas Comunidades $\mathrm{Au}$ tónomas, como lo prueba el artículo de Cirera y colaboradores ${ }^{4}$ que se publica en este mismo número de la revista, y en el que se demuestra la eficacia y la factibilidad de los talleres de aprendizaje de la certificación, tanto en el ámbito de la formación de pregrado como de la formación continuada de los profesionales de la medicina. Experiencia que, además, se valora como satisfactoria por parte de la mayoría de los participantes.

Lo cual puede considerarse como un ejemplo de la responsabilidad de los sanitarios para mejorar la calidad de las estadísticas de mortalidad, mejora que podría verse incrementada si se aprovechara la oportunidad de recuperar los trabajos del grupo mencionado anteriormente.

Paralelamente, la Sociedad Española de Epidemiología ha constituido un grupo de trabajo sobre la décima revisión, que presentará en un informe en la próxima reunión de la Sociedad el próximo mes de octubre en Sevilla 5 .

Precisamente, la entrada en vigor de la décima revisión podría constituir un estímulo para que tanto las autoridades sanitarias centrales como las autonómicas, reemprendieran el trabajo desarrollado hace ya casi diez años y, junto con otras iniciativas como la reciente creación del Centro Nacional de Información Sanitaria en el seno del Instituto de Salud Carlos III y el establecimiento del Indice Nacional de Defunción, comportaran una nueva mejora de la calidad de las estadísticas oficiales de mortalidad, las cuales siguen siendo imprescindibles para llevar a 
cabo adecuadamente los trabajos de planificación y de evaluación sanitaria que nuestro sistema nacional de salud requiere.

\section{BIBLIOGRAFÍA}

1. Lilienfeld AM [editor] Times, places, and persons. Aspects of the history of Epidemiolngy. Baltimore: The Johns Hopkins University Press; 1978. p. 28-39.

2. Nadal J. La Población Española. Barcelona: Ariel; 1976. p. 129.
3. Plasencia A. Las estadísticas de mortalidad perinatal en España: ¿Hay que tirar la toalla? [editorial]. Gac Sanit 1990: 4: 91.

4. Cirera Ll, Martínez C, Contreras J, Navarro C. Aprendizaje y satisfacción de los talleres de pre y postgrado de Medicina para la mejora de la certificación de las causas de defunción. Rev Esp Salud Pública 1998: 72: 185-195.

5. Cirera L1, Vázquez E (eds). La implantación de la 10 . $^{\text {a }}$ revisión de la Clasificación Internacional de Enfermedades en España. Sevilla: Socicdad Española de Epidemiología; 1998. 